\title{
31 Pflanzengifte in natürlichen Lebensmitteln
}

(c) Springer-Verlag GmbH Deutschland, ein Teil von Springer Nature 2018

D. Mathias, Fit und gesund von 1 bis Hundert

https://doi.org/10.1007/978-3-662-56307-6_31

Anders als die von Menschen versprühten Pflanzenschutzmittel ( $\$$ Kap. 30) enthalten Pflanzen oft auch eigene Gifte. Diese Phytotoxine gehören zu den unerwünschten Bestandteilen in unserer natürlichen Nahrung. Klassische Beispiele sind Solanin in grünen Teilen von Tomaten oder in rohen Kartoffeln, Phasin in rohen Bohnen, Blausäure in Aprikosenkernen oder Cumarin im Zimt. Interessant sind ferner Sambunigrin im Holunder, Anthrachinon und Oxalsäure in rohem Rhabarber und Nikotin in grünen Teilen der Aubergine. Manche Kräuterteesorten sind mit den krebserregenden Pyrrolizidinalkaloiden belastet - kein starkes Gift, aber auch problematisch ist Morphin im Mohn.

Phasine sind Lektine und gehören zur Gruppe der Hämagglutinine. Sie beeinflussen die Funktion unserer roten Blutkörperchen negativ. Eine bestimmte Menge roh verzehrter Bohnenarten bewirkt Symptome wie blutiges Erbrechen und Durchfall sowie Magen- und Darmbeschwerden. Ist die letale Dosis nicht erreicht, verschwinden die Symptome in der Regel bereits nach wenigen Stunden. Durch viertelstündiges Kochen werden die Phasine zerstört.

Glycyrrhizin gehört zu den Saponinen. Es schmeckt süß, während alle anderen Saponine einen bitteren Geschmack auslösen. Glycyrrhizin ist u. a. der Geschmacksstoff der Lakritze und kommt in der Wurzel der Süßholzpflanze vor. Hohe Dosen von mehr als $100 \mathrm{mg}$ pro Tag steigern den Blutdruck, haben hämolysierende Wirkungen und hemmen die Wasserausscheidung. Ein mäßiger Verzehr von Lakritze ist für gesunde Personen unproblematisch. Menschen mit Bluthochdruck oder Herz-Kreislauf-Patienten sollten beim Lakritzeverzehr Zurückhaltung üben.
Blausäure findet sich in den Kernen von Rosengewächsen wie Aprikose, Bittermandel, Pfirsich oder Kirsche. Beginnende Symptome nach zu hohem Verzehr sind Rotfärbungen der Haut und stoßweises Atmen. Für Kinder können bereits 5-10 Bittermandeln tödlich sein.

Cumarin kommt im Zimt, aber auch in Datteln vor. Wenn von diesen zu viel verzehrt wird, können Schwindel, Kopfschmerzen, Müdigkeit und Übelkeit die Folge sein.

Tee, Kakao und Schokolade enthalten in geringen Mengen Oxalsäure. Größere Mengen davon kann der Rhabarber enthalten. Die Aufnahme von zu viel Oxalsäure führt zu Symptomen wie Krämpfe, Erbrechen und Kreislaufkollaps. Oxalsäure bildet mit Calcium schwerlösliche Salze, die in größerer Menge zu Nierenschädigungen führen.

Pyrrolizidinalkaloide kommen weltweit in sehr vielen Pflanzenarten vor. Sie werden von diesen zur Abwehr von Fressfeinden gebildet. Der Schaden beim Menschen entsteht nicht direkt durch die Alkaloide selbst, sondern durch deren Abbauprodukte in der Leber. Diese können Auslöser für Krebserkrankungen sein und das Erbgut schädigen. Pyrrolizidinalkaloide finden sich hauptsächlich in verschiedenen Kräutertees.

Viele dieser Pflanzengifte lassen sich leicht schon durch einfaches Abkochen zerstören oder zumindest aus der Pflanze entfernen, Solanin z. B. aus den grünen Stellen von Tomaten und Kartoffeln. Bei Erwachsenen kommt es in unserer heutigen aufgeklärten Zeit nur noch selten zu Vergiftungen durch Beeren oder Pflanzen (Lüde et al 2016). Gefährdet sind hier eher größere Kinder, die gerne mal probieren und leichter auch Verwechslungen erliegen. 